\title{
Erratum: Limits to magnetic quantum criticality from nuclear spins [Phys. Rev. B 103, 064405 (2021)]
}

\author{
Heike Eisenlohr $\odot$ and Matthias Vojta \\ (Received 2 June 2021; published 10 June 2021)
}

DOI: 10.1103/PhysRevB.103.219902

We clarify that the shift of the quantum phase transition in the transverse-field Ising model by hyperfine coupling to nuclear spins has been discussed in previous literature [1-4] in the context of $\mathrm{LiHoF}_{4}$ as has the presence of a hybridized soft mode [4-6]. Our results are consistent with these earlier works. However, neither the scaling of the phase-transition shift with the strength of the hyperfine coupling nor the general phenomenology of magnetic quantum criticality in the presence of nuclear spins were considered before the publication of our paper.

[1] D. Bitko, T. F. Rosenbaum, and G. Aeppli, Phys. Rev. Lett. 77, 940 (1996).

[2] P. B. Chakraborty, P. Henelius, H. Kjønsberg, A. W. Sandvik, and S. M. Girvin, Phys. Rev. B 70, 144411 (2004).

[3] S. M. A. Tabei, M. J. P. Gingras, Y.-J. Kao, and T. Yavors'kii, Phys. Rev. B 78, 184408 (2008).
[4] R. D. McKenzie and P. C. E. Stamp, Phys. Rev. B 97, 214430 (2018).

[5] H. M. Ronnow, R. Parthasarathy, J. Jensen, G. Aeppli, T. F. Rosenbaum, and D. F. McMorrow, Science 308, 389 (2005).

[6] I. Kovacevic, P. Babkevich, M. Jeong, J. O. Piatek, G. Boero, and H. M. Rønnow, Phys. Rev. B 94, 214433 (2016). 